\title{
A monitoring system for Cold Chain Logistics Vehicle based on Beidou Satellites
}

\author{
Yang Kai ${ }^{1, a}$, Zhang Xiaofeng ${ }^{2, b}$, Shi Quan ${ }^{2, c, *}$ and Sun Ling ${ }^{1, d}$ \\ ${ }^{1}$ School of Electronics and Information, Nantong University, Seyuan Road No.9, Nantong, China \\ ${ }^{2}$ School of Transportation, Nantong University, Seyuan Road No.9, Nantong, China \\ ayk504160278@hotmail.com, ${ }^{\mathrm{b}}$ 2846870147@qq.com, ${ }^{\mathrm{c}}$ sq@ntu.edu.cn, ${ }^{\mathrm{d}}$ sun.1@ntu.edu.cn \\ *corresponding author
}

Keywords: Beidou, Logistics vehicle, Monitoring system, Si4432.

\begin{abstract}
In food transport process by cold-chain, the monitoring of temperature and humidity is very important. In order to strengthen the supervision of the cold chain logistics industry, improve the reliability of the cold chain logistics vehicle monitoring system and the safety of cold-chain food during transportation, a cold chain logistics vehicle monitoring system is designed and realized, which is based on Beidou satellites. The designed system can obtain not only the position but also the temperature and humidity information of the vehicle by the Beidou positioning module and the sensor acquisition sub-net. The obtained information then is transmitted to the monitoring center through the GPRS network. Therefore, the supervisor can get the current temperature and humidity information, find the location of the vehicle on the map by opening related web pages or the APP in mobile phone. If the value of the temperature or humidity exceeds the set threshold, alarm is generated. Practical tests prove that the proposed system can monitor the temperature and humidity information effectively and reliably, show the path of logistics vehicle, which denote that data communication is stable and reliable. With the independent Beidou technology, the system is convenient to use and has the wide range of applications, which conforms to the development of the Internet of things and the commercialization of Beidou technology.
\end{abstract}

\section{Introduction}

With the demand of satellite position increasing widely for industrial application in our daily life, the commercial development of global satellite positioning system is growing quickly. Beidou Position System which is developed in China is pushing for the application ${ }^{[1-3]}$. The commercial of Beidou satellites will effectively address the needs of the market, and the cold chain logistics vehicles' location monitoring is a typical example. As the development of China's urbanization and the improvement of people's living standard, the concern of cold chain transportation turns to be a problem, protecting the quality and safety of the food needs to focus more ${ }^{[4-5]}$. In order to achieve effective monitoring of cold chain logistics vehicles, strengthen monitoring the temperature and 
humidity of cold chain logistics during the transport process, also promote the development of the Beidou industry, a monitoring system for cold chain based on Beidou is designed in this paper.

The designed system consists of four parts: a set of on-board humiture measurement nodes, the master node, the monitoring center server and mobile phone application. It can effectively send the track of the cold chain vehicle and humiture information inside the vehicle to a remote monitoring center in time, which is convenient for management to check or verify. At the same time, this system also has the alarm system, when the cold chain vehicle's temperature or humidity deviates from its threshold, the system will alarm up to remind the driver and also send information to the center administrator, ensuring the quality and safety of the cold chain food effectively during transportation. The design and implement of this system are detailed as follows.

\section{Overall}

The architecture of the whole system is shown in Fig.1, while Fig.2 shows the inner connection of the cold chain Vehicle. It can be seen that the acquisition nodes and the master node make up the information collecting network of the cold chain vehicle. The master node also responsible for the communication with GPRS and Beidou satellites. This design contains multiple temperature and humidity acquisition nodes, Si4432 wireless communication module, data aggregation and retransmission node, Beidou positioning module, GPRS data communication module, monitoring center server and mobile phone client.

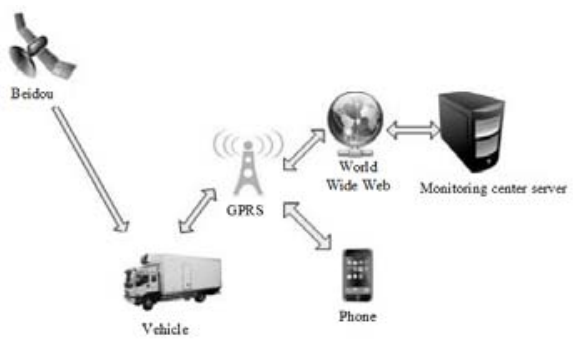

Figure 1 System architecture

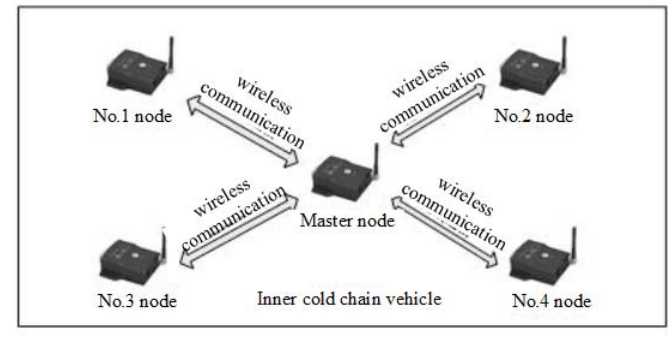

Figure 2 Internal structure of cold chain vehicle

Four temperature and humidity acquisition nodes and a master node are assembled for each cold chain logistics vehicle, and they construct the information acquisition sub network, each slave node communicate with the master node by wireless transceiver Si4432, the master node equipped with Beidou positioning module and GPRS communication module. For the whole system, the slave nodes are responsible for collecting temperature and humidity date at different places in the cold chain vehicle, then transfer the data to the master node, the master control node receives each slave node's humiture information and converge them together along with the Beidou positioning information. Then these integrated data will be sent to the monitoring center server system through GPRS network at regular intervals. When the user logs on the monitoring center server, the related web pages will present the current temperature and humidity of the logistics vehicle, and display the location of the vehicle on the map. These information can also synchronized to user's mobile client, users can see the corresponding information by the mobile application, when the temperature and humidity deviate from the threshold, the corresponding alarm reminder will appear on the phone.

\subsection{Hardware Design}

This system uses STC12C5A60S2 as the main microcontroller, combined with GPRS module, Beidou module, humiture acquisition module and Si4432 wireless communication module, realizing 
the cold chain logistics vehicle monitoring system based on Beidou satellite positioning technology. The hardware block diagrams are shown in Fig.3.

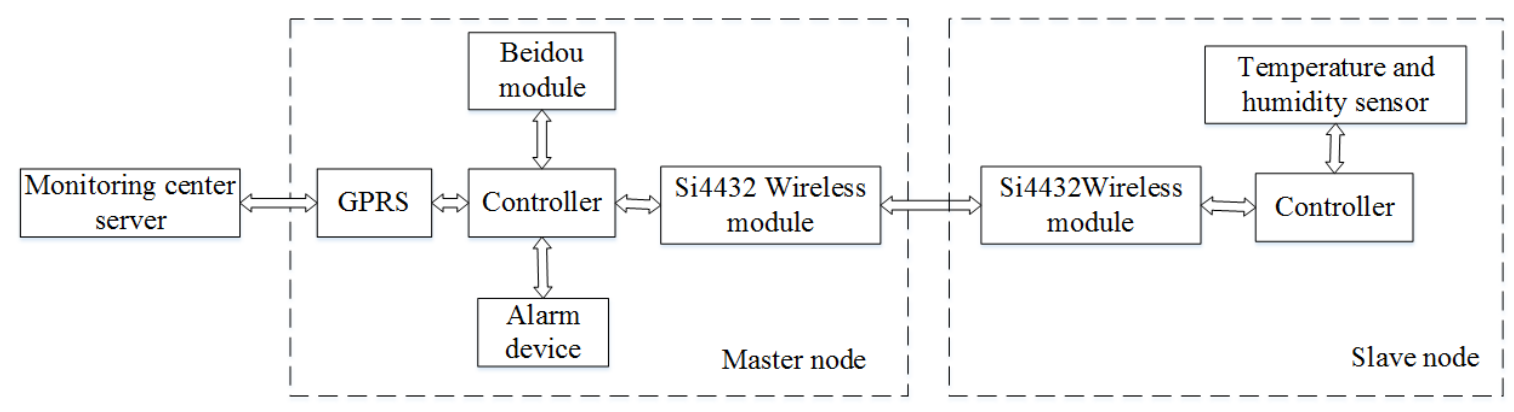

Figure 3 Hardware system diagram

In Fig.3, UM220-III N Dual system high performance GNSS module is used for positon, this module supports BD2 B1 frequency point, its tracking sensitivity is about $-160 \mathrm{dBm}$, capturing sensitivity is $-145 \mathrm{dBm}$. The location information acquired by this module can be get through UART interface communication. The module operating voltage range is $2.7 \sim 3.3 \mathrm{~V}$, it' $\mathrm{s}$ easy to integrate with the whole system without any power conversion. Most importantly, its working temperature is around $-40^{\circ} \mathrm{C} \sim 80^{\circ} \mathrm{C}$, so it is capable of cold chain logistics vehicle, even put it inside the vehicle.

GPRS data communication module used is HUAWEI EM310, this module embedded TCP/IP protocol, support data, voice, SMS and fax communication. It is connected to the master controller by UART interface, realizing the data transmission between the master node and the monitoring center. In order to access the GPRS network, it is necessary to use some relative AT commonds to establish the network connection between EM310 and GPRS network.

For temperature and humidity acquisition, Si4432 modules are used for wireless sub-net. This module operates at the band of $433 \mathrm{MHz}$, it's free to use in China, because it is in the ISM band which can be directly used (industrial, scientific and medical band). The output power of Si4432 is up to $+20 \mathrm{dBm}$, receiving sensitivity is $-121 \mathrm{dBm}$, these ensure the excellent transmission link, and suitable for temperature and humidity data transmission inside the cold chain vehicles.

The actual master node components are shown in Fig. 4.

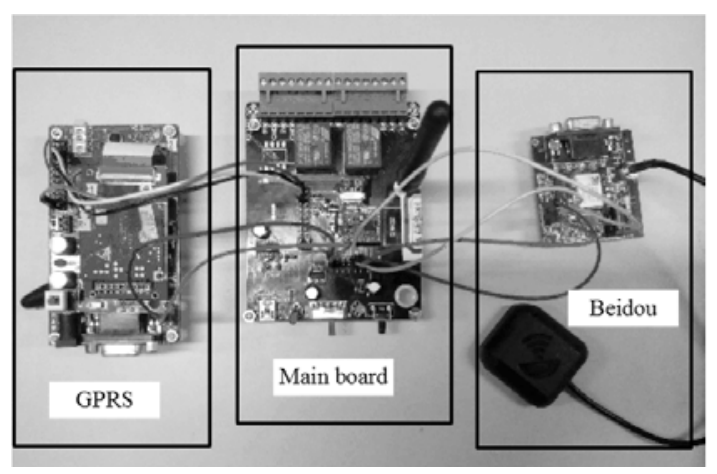

Figure 4 Hardware of Master node

\subsection{Software Design}

\subsubsection{Master node program}

The master node controller first use UART_Init(), BEIDOU_Init(), GPRS_Init() and RF4432_Init() functions to initialize serial port, Bēidou, GPRS and Sï4432 module, the position 
information received by the Beidou module will be temporarily stored in the main controller, then use send_data(AT_cmd[7],dat) function to deliver the needed information to the monitor center. The frequency of sending the data is setup by the function delay(). Location information of the vehicle is sent every 10 seconds, and followed are temperature and humidity data. The time interval of the transmission can be determined according to the specific circumstances. For fear of data exception caused by system failure, self- detection mechanism is added. If an error occurs, the error message will be sent to the server and notify the administrator. Fig. 5 is the program flow chart of the main control node.

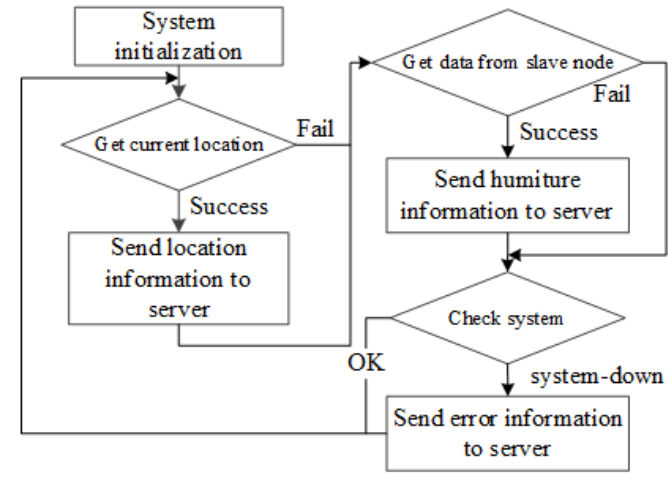

Figure 5 Program flow of Master node

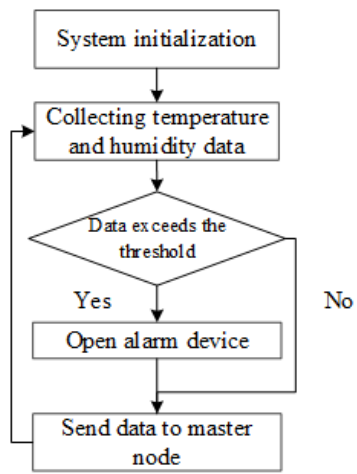

Figure 6 Program flow of Salve node

\subsubsection{Slave node program}

Acquisition node first use RF4432_Init() and SHT_Init() functions to initialize the Si4432 wireless transmission module and temperature and humidity collecting module respectively, Then store the collected the temperature and humidity information in a array waiting to send. When the data preparation is ready, the controller will call the send function RF4432_TxPacket() transferring data to the master node. Fig. 6 shows the program flow chart of acquisition node.

\subsection{Web presentation and Android client}

During the transportation of cold chain logistics vehicles, temperature and humidity exceed the threshold, the warning reminder can notice the carrier to fix the problem, or call the background service center for help. But if the transport personnel failed to discover and solve the problem, or on the wrong way to the destination, speeding or some other abnormal situations, the background need to know these latest information. Therefore, this paper developed an Android client and web application, managers can obtain the temperature and humidity information, see the vehicles of realtime geographic location, license plate and vehicle speed information in the transport process. When the temperature and humidity exceeds the threshold, the mobile phone will alarm to remind the transportation officer.

\subsubsection{Web Presentation}

In web application, the user need to login first, the user name and password are used for authentication. After certification, the page will jump to Fig. 7, displaying vehicle location information. This interface shows the current cold chain logistics vehicle number and license plate number, click on the license plate number, the interface will display the cold chain vehicle position, temperature and humidity information corresponding to the license plate. In addition, the user can click on details button to view the change curve of temperature and humidity, as shown on Fig. 8. 


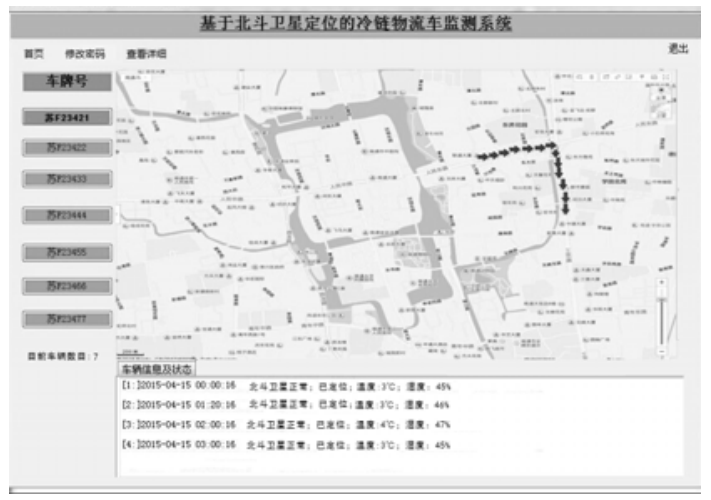

Figure 7 Web display of Vehicle

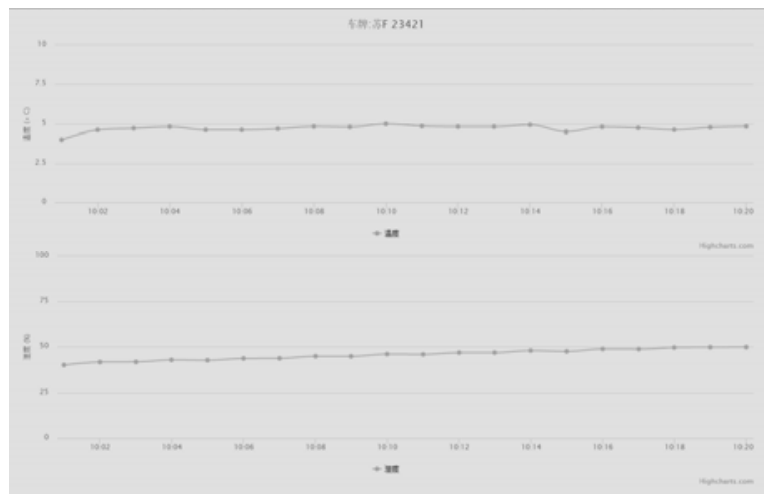

Figure 8 Temperature and humidity monitoring

\subsubsection{Android Client}

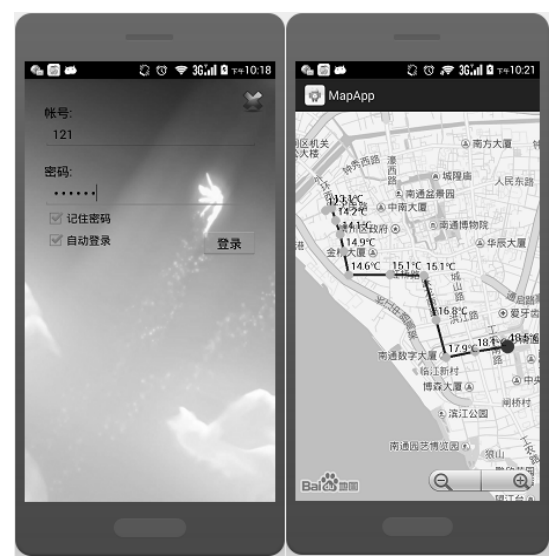

Figure 9 Login interface and Display of vehicle location on mobile phone

Android client receives data from the server, extracts the temperature, humidity, latitude and longitude information and displays in real-time. When users open the application, it will turn out like Figure 9. Typing in the user name and password to login in. The home page lists all the cold chain logistics vehicles. Click one of the list items can view the details of the selected cold chain logistics vehicle. Click the map can see the location of the vehicle. And the Android application will redraw in the map at overlay layer, connecting the points of the vehicle position to form the route curve of vehicle, like Fig. 9. The client can also calculate the average speed of the cold chain logistics vehicle in a certain distance according to the formula speed $=$ distance $/$ time. If there is a moment when the temperature and humidity of the cold chain logistics vehicle exceeds the preset temperature and humidity value, the text messages will turn up, reminding the user to deal with the problem in a timely manner.

\section{System testing and analysis}

The system is tested under normal temperature for one week to make sure the stability of data transmission. The result turns to be nice, the system works stably, and the temperature and humidity information measured by each sub node can react in real time to the web end, slave nodes data is not lost, and no system crash.

In addition, the actual positioning ability is also tested, the location in the map is close to reality, Web and mobile terminal display the same path as the actual path, the positioning information is reliable. 
Due to the use of Si4432 and GPRS, these wireless communication technology make the hardware installation very convenient, avoid the complexity of physical wiring, easy to use and easy to maintain.

The application of Web and mobile terminal can display the route of cold chain logistics vehicle in real time, how much time is the vehicle spent on the way and its inner temperature and humidity. Meanwhile, the alarm function effectively reducing the cold chain logistics management costs, improving its efficiency.

\section{Conclusion}

In this paper, the designed cold chain logistics vehicle monitoring system based on Beidou satellite can effectively monitor the temperature, humidity and position information of the vehicle, the information can be transmitted to the monitoring center in real time. The practical application shows that the system is stable and reliable, having strong compatibility and expansibility, also can be used for monitoring the position information of moving objects in various industries. The system not only promote the development of China's cold chain logistics industry, but also help accelerate the commercialization of Beidou.

\section{Acknowledgements}

This research has been partially supported by Graduate Student Innovation Program, Project Number YKC15014. The colleges and universities innovation training provincial key projects of Jiangsu Province (201610304039Z).

\section{References}

[1] $\mathrm{Hu} \mathrm{C}$, Tian Y, Yang X, et al. Background Ionosphere Effects on Geosynchronous SAR Focusing: Theoretical Analysis and Verification Based on the BeiDou Navigation Satellite System (BDS) [J]. IEEE Journal of Selected Topics in Applied Earth Observations and Remote Sensing, 2016, 9(3): 1143-1162.

[2] Xiao W J, Zhang F Z, He H X. Application Analysis of IOT Based on Beidou Navigation System[C]//Advanced Materials Research. Trans Tech Publications, 2013, 718: 2401-2408.

[3] Ding L, Chen Y. Advantage of the Beidou Navigation System: Application in Emergency Logistics[C]//Instrumentation \& Measurement, Computer, Communication and Control (IMCCC), 2016 Sixth International Conference on. IEEE, 2016: 439-442.

[4] Aung M M, Chang Y S. Traceability in a food supply chain: Safety and quality perspectives [J]. Food control, 2014, 39: 172-184.

[5] Lu L, Zheng W, Lv Z, et al. Development and application of time-temperature indicators used on food during the cold chain logistics[J]. Packaging Technology and Science, 2013, 26(S1): 80-90. 